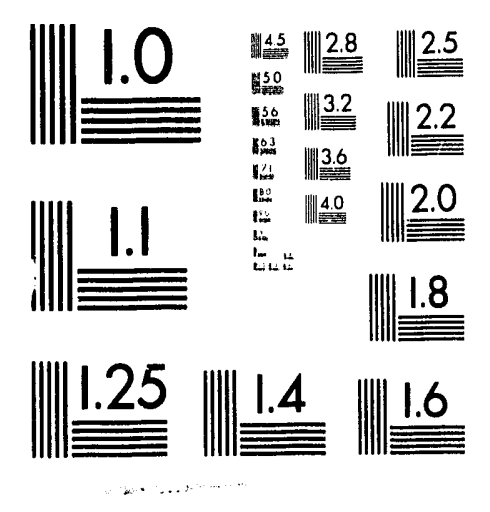



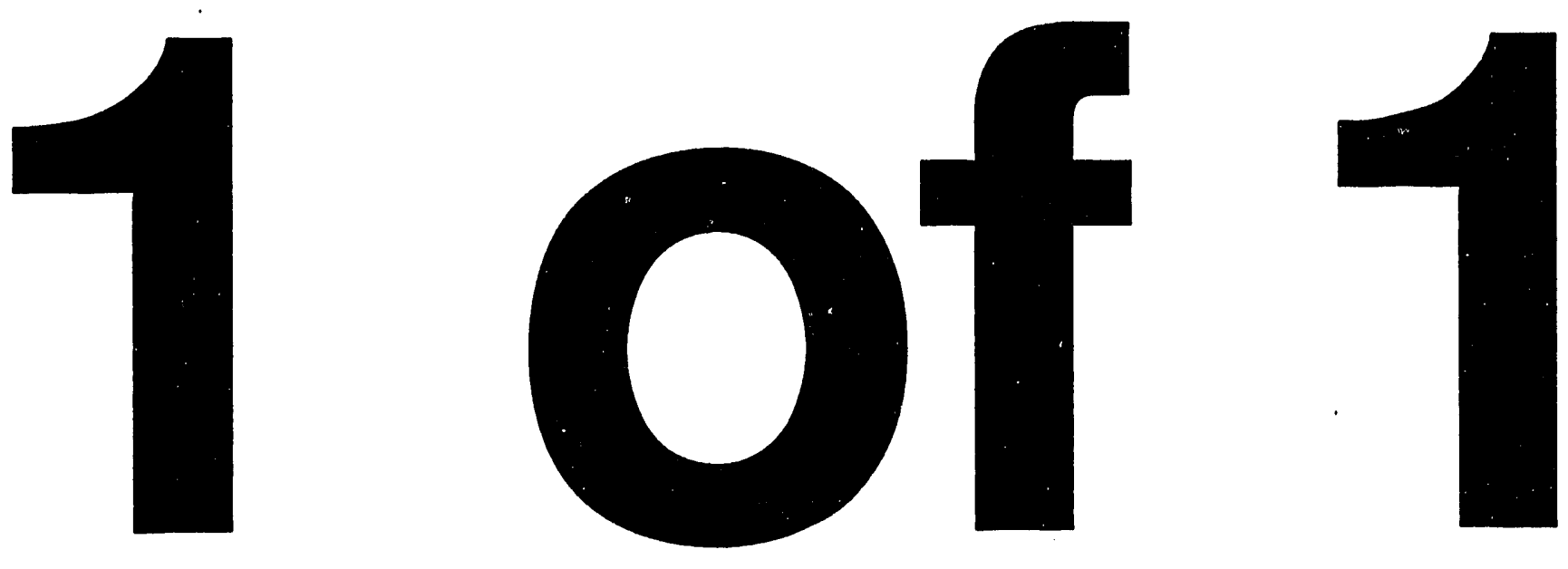
SAND93-1763

Unlimited Release

Distribution

Printed August 1993

UC-700

\title{
A Method to Simulate Viscous Diffusion of Vorticity by Convective Transport of Vortices at a Non-Solenoidal Velocity
}

\author{
S. N. Kempka and J. H. Strickland \\ Engineering Sciences Center \\ Sandia National Laboratories
}

Albuquerque, NM 87185

\begin{abstract}
A numerical method to simulate viscous diffusion of vorticity using vortex blobs (i.e., without a grid) is presented. The method consists of casting the effects of viscous diffusion into an effective "diffusion velocity" at which vortex blobs convect. The diffusion velocity was proposed previously by Ogami and Akamatsu', but they did not consider the effects of the divergence of the diffusion velocity. In fact, the diffusion velocity is highly non-solenoidal, which significantly affects the area over which a vortex blob diffuses. A formulation is presented that relates the area expansion to the diffusion velocity divergence. By taking into account the area expansion, more accurate simulations of diffusion are obtained, as demonstrated by a comparison of numerical and analytical diffusion solutions. Results from simulations show that vortex areas expand significantly in regions of large vorticity gradients. As a result of the area expansion, adjacent vortices remain overlapped, thereby maintaining smooth solution fields. The non-solenoidal diffusion velocity method is easily implemented in vortex blob algorithms, thus facilitating the development of vortex methods to simulate flows with finite Reynolds numbers.
\end{abstract}


This page is intentionally blank. 


\section{Table of Contents}

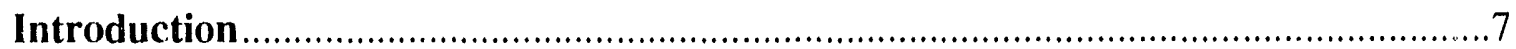

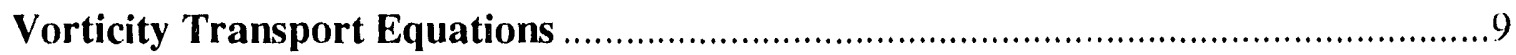

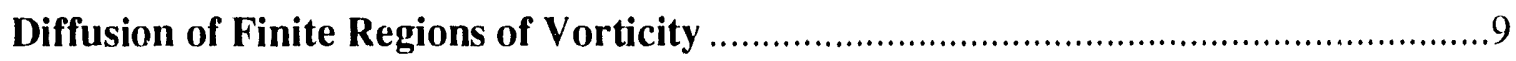

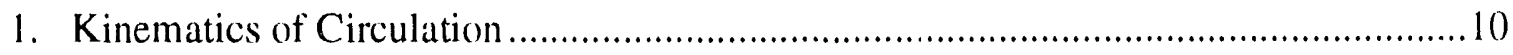

2. Inclusion of Dynamics within the Kinematics of Circulation .................................

2.1 Inviscid Transport in Terms of Circulation.............................................. 11

2.2 Viscous Transport in Terms of Circulation............................................. 11

2.3 Determination of the Diffusion Velocity ................................................ 11

3. Effects of Non-Solenoidal Velocity Fields .................................................... 13

Implementation of the Non-Solenoidal Diffusion Velocity Method ..........................14

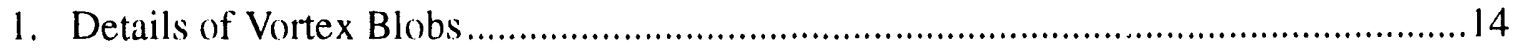

1.1 Calculation of Diffusion Velocity and Its Divergence..................................15

1.2 Implementation of the Diffusion Velocity Divergence................................. 16

2. Summary of the Diffusion Velocity Vorticity Formulation ..................................... 17

Evaluation of Accuracy of the Diffusion Velocity Technique................................... 18

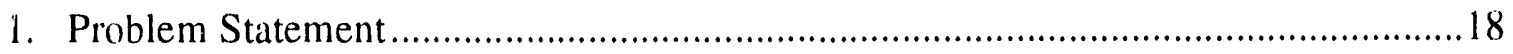

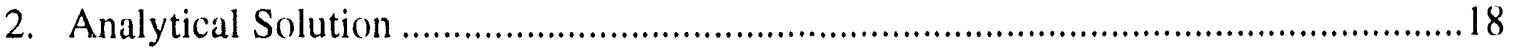

3. Diffusion Velocity Simulations of Pure Diffusion ............................................. 18

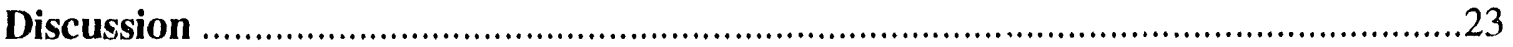

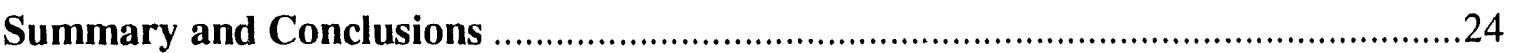

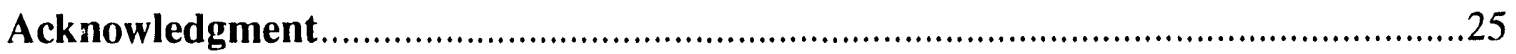

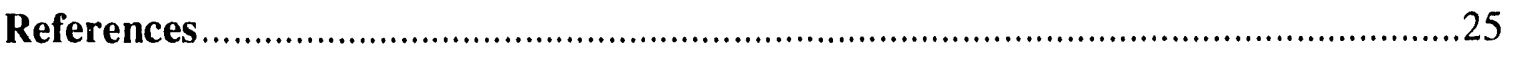

APPENDIX A: Formulas for a Two-Dimensional Vorticity Field ..............................27

APPENDIX B: Formulas for a One-Dimensional Vorticity Field..............................28 
This page is intentionally blank. 


\section{Introduction}

The use of discrete vortex methods to simulate finite Reynolds number flows lags considerably behind grid-based methods, such as finite element techniques. A principal reason for this disparity is the difficulty of accurately including viscous diffusion, which is the topic addressed herein.

The difficulty of including viscous diffusion in vortex methods arises from the fact that discrete vortex methods are based on inviscid flow theory. As a result, viscous diffusion is treated as a secondary, but important, effect within the framework of vortex methods. The most common approach is to use operator splitting in which inviscid and viscid transport are treated in sequential steps. In the most commonly used operator splitting scheme, the implementation of diffusion is reduced to solving the diffusion equation, which is a relatively simple procedure if a grid is available. Vortex methods, however, do not require a grid to simulate inviscid transport, so a griu is not available. Moreover, there is significant motivation to avoid the use of grids since their construction can require significant time and effort, especially for complex geometries.

Since vortex methods are Lagrangian, it would be more consistent to implement diffusion in a Lagrangian algorithm. Several such approaches have been proposed, including the Gaussian random walk method ${ }^{3}$, the diffusing core method $^{6}$, the method by Fishelov ${ }^{4}$, and the diffusion velocity method by Ogami and Akamatsu?

The Gaussian random walk method was introduced by Chorin ${ }^{3}$, and is based on the relationship between random walks and diffusion. For a field of particles moving with Gaussian random walks, the probability of a particle being at a particular location is specified by the diffusion equation. Thus, adding a random walk to the motion of vorticity particles simulates diffusion in a statistical sense. A vortex blob represents a finite region of vorticity (many pointwise particles of vorticity), however, so that moving a vortex blob with a Gaussian random walk specifies incorrectly that all the particles within a blob move with the same random walk. As a result, the random walk method applied to vortex blobs only approximates the diffusion of vorticity in the mean. This approximation is believed to be the origin of the weli-known limitation that the random walk method is appropriate only for Reynolds numbers of $\mathrm{O}\left(10^{2}\right)$ or greater. Several large Reynolds number flows have been successfully simulated using the random walk approach, such as flow over a backward facing step ${ }^{10}$. The solutions are "jittery," however, since statistically well-converged, smooth solutions require an intractable number of vortices.

As mentioned above, the large Reynolds number restriction of the random walk method is believed to arise from the fact that the random walk is added to the motion of finite regions of vorticity, rather than to each infinitesimal point of vorticity. This approximation is necessary to make the method computationally feasible, but omits an important feature of diffusion which is emphasized in the present analyses, namely that the volume occupied by a particular set of particles increases.

The diffusing core method ${ }^{6}$ accounts for the expansion of the volume occupied by a region of vorticity, but in a manner that does not depend on the rest of the vorticity field. Ad- 
ditionally, there is no motion of the vortex center, and the combined equations associated with inviscid convection and the diffusing core algorithm are not the Navier-Stokes equations. 5 . As will be shown, the formulation developed here indicates that the diffusing core method is appropriate only for a domain containing a single vortex. It will also be shown that for more than one vortex, viscous interactions between vortices yield a translation and an expansion of each vortex.

Ashurst ${ }^{1}$ accounted for viscous effects in a vortex simulation of a mixing layer by combining the random walk and diffusing core methods. Saffman and Baker suggest that this amounts to counting the effects of viscosity twice. However, in the derivation of the diffusion model to be presented, it is shown that both translation and expansion of vortices are necessary to describe viscous diffusion. Thus, Ashurst's approach is in fact conceptually appropriate, although translation by random walks and expansion by the diffusing core method both have significant limitations. The proposed model provides improved alternatives for both diffusive translation and expansion. A deterministic diffusion velocity is used instead of random walks, and core expansion is prescribed by the diffusion velocity divergence instead of the diffusing core method. The proposed approach takes advantage of two recent developments which are discussed below.

The method proposed by Fishelov ${ }^{4}$ essentially involves the calculation of vorticity fluxes from one vortex to another. Vorticity fluxes are based on the vorticity gradient that is calculated by differentiating the assumed vorticity distribution of each vortex. This approach is appealing because it is consistent with other aspects of vortex methods. However, the transport of vorticity into regions where vortices do not exist requires that new vortices be added continually during a calculation. The proliferation of vortices can be treated with a vortex merging technique, but vortex merging does not preserve linear or angular momentum, and such errors are not desirable, even though they often have negligibly small effects.

The diffusion velocity method by Ogami and Akamatsu ${ }^{7}$ essentially casts diffusion as a convective process using a diffusion velocity. Unlike the random walk method, the motion specified by the diffusion velocity is deterministic, and therefore statistical convergence issues are not a concern. But, similar to the random walk method, the diffusion velocity method neglects vortex expansion. Nonetheless, the concept of a diffusion velocity is appealing since it can be easily implemented within vortex methods.

The starting point for the present analyses is the diffusive velocity method proposed by Ogami and Akamatsu, using Fishelov's method to represent spatial derivatives of vorticity. We present a method in which vortices convect at the diffusion velocity and the blob sizes change with time according to the divergence of the diffusion velocity. We refer to this approach as the non-solenoidal diffusion velocity method to emphasize the significance of the diffusion velocity divergence. Derivation of the method is based largely on the kinematics of circulation and is shown to be accurate by comparing its results with analytical diffusion solutions. 


\section{Vorticity Transport Equations}

The vorticity form of the three-dimensional Navier-Stokes equations for a constant density (and therefore incompressible) flow with constant viscosity, $v$, is

$$
\frac{\partial \vec{\omega}}{\partial t}+(\vec{u} \bullet \nabla) \vec{\omega}=(\vec{\omega} \bullet \nabla) \vec{u}+v \nabla^{2} \vec{\omega}
$$

Vorticity is defined as the curl of the velocity field,

$$
\vec{\omega}=\nabla \times \vec{u},
$$

and the incompressibility constraint is,

$$
\nabla \cdot \vec{u}=0 \text {. }
$$

If the velocity normal to the boundary is specified, Eqs. (1), (2), and (3) form a closed system that allows the vorticity field and the velocity field to be determined.

\section{Diffusion of Finite Regions of Vorticity}

Before considering diffusion as a convective process, details of viscous diffusion are reviewed in terms of a finite volume of vorticity. We choose the three-dimensional volume element to be a cylinder whose two end faces are material surfaces with vector area $A^{2}$. The integral of vorticity over the area $A$ is referred to as the circulation, $\Gamma$,

$$
\Gamma=\int_{A} \vec{\omega} \bullet \hat{n} d A .
$$

If every point in the area $A$ is assumed to convect at the local fluid velocity, the circulation varies with time as ${ }^{2}$,

$$
\frac{d \Gamma}{d t}=v \int_{A} \nabla^{2} \vec{\omega} \bullet \hat{n} d A,
$$

where the viscosity, $v$, is assumed to be constant, as is the fluid density. The change in circulation indicated by Equation (5) is due to the diffusion of vorticity across the boundary of the fluid area. As shown in Figure 1, a region of vorticity initially bounded within in a fluid area convects and expands beyond the fluid area as a result of diffusion.

The objective of the non-solenoidal diffusion velocity approach is to re-define the area in the circulation integral in such a way that it remains coincident with a particular region of vorticity. In order to develop this approach, we first consider the kinematics of circulation. 


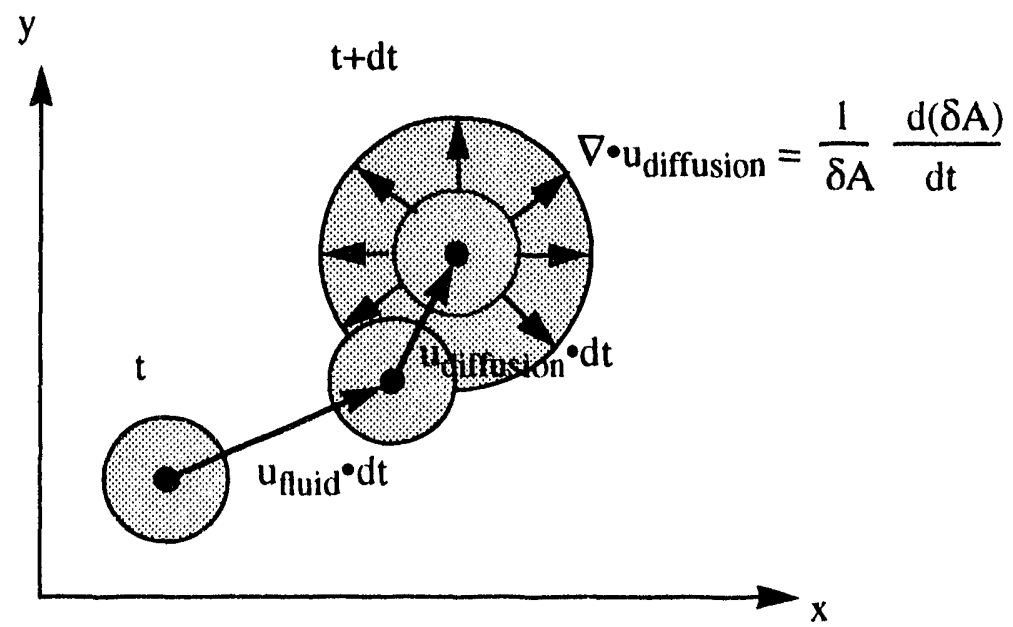

Figure 1 Inviscid and viscous transport of a region of vorticity. Inviscid transport is shown by the translation of the vorticity at the incompressible fluid velocity, with no change in the area $\mathrm{A}$ occupied by the vorticity. Viscous transport is shown by additional translation and expansion of the area occupied by the vorticity. The fixed shape of the regions omits possible effects of shear on the vorticity field, which can be remedied through the use of adaption techniques not described here.

\section{Kinematics of Circulation}

The circulation of a cylindrical fluid element with end face area $A$ is

$$
\Gamma=\int_{A} \vec{\omega} \bullet \hat{n} d A
$$

If every point in the area $A$ is assumed to convect at an arbitrary velocity $\vec{u}_{a}$ (i.e., the relation $\nabla \times \vec{u}_{a}=\vec{\omega}$ need not be true), then the time-derivative of the circulation is

$$
\frac{d_{a} \Gamma}{d t}=\int_{A}\left[\frac{\partial \vec{\omega}}{\partial t}+\left(\vec{u}_{a} \bullet \nabla\right) \vec{\omega}+\vec{\omega}\left(\nabla \bullet \vec{u}_{a}\right)-(\vec{\omega} \bullet \nabla) \vec{u}_{a}\right] \bullet \hat{n} d A
$$

where the notation $d_{a} / d t$ denotes differentiation of a region convecting at an arbitrary velocity. Lack of a subscript implies differentiation of a region convecting at the local fluid velocity $\vec{u}$, for which $\nabla \times \vec{u}=\vec{\omega}$. The latter two terms of the integrand arise from the time-derivative of the end face area $A$.

\section{Inclusion of Dynamics within the Kinematics of Circulation}

Vorticity dynamics in a viscous flow can be thought of as occurring in two simultaneous steps: inviscid dynamics, and viscous dynamics. Inviscid dynamics are governed by 


$$
\frac{\partial \vec{\omega}}{\partial t}+(\vec{u} \bullet \nabla) \vec{\omega}=(\vec{\omega} \bullet \nabla) \vec{u}
$$

Viscous dynamics are governed by

$$
\frac{\partial \vec{\omega}}{\partial t}=v \nabla^{2} \vec{\omega}
$$

\subsection{Inviscid Transport in Terms of Circulation}

First, we re-state the well-known theorem of Helmholtz that inviscid vorticity convects at the local fluid velocity and the circulation of a region of vorticity remains constant. This can be obtained within the present framework by assuming $\vec{u}=\vec{u}_{o}$, and substituting the inviscid vorticity equation Eq. (8) into the general kinematic equation for circulation Eq. (7). The well-known result is

$$
\frac{d \Gamma}{d t}=0
$$

\subsection{Viscous Transport in Terms of Circulation}

For viscous transport, we assume that the area in the circulation integral convects at a yet to be determined diffusion velocity $\vec{u}_{a}=\vec{u}_{d}$, so as to remain coincident with the area occupied by a particular sub-field of vorticity (a vortex blob) as it diffuses. In this way, we can explicitly track a particular region of vorticity. Additionally, since vorticity can be neither created nor destroyed in the interior a homogeneous fluid (but can rotate, stretch, or compress, as can the fluid area), the circulation of such a region cannot change. This is specified by,

$$
\frac{d_{d} \Gamma}{d t}=\int_{A}\left[\frac{\partial \vec{\omega}}{\partial t}+\left(\vec{u}_{d} \bullet \nabla\right) \vec{\omega}+\vec{\omega}\left(\nabla \bullet \vec{u}_{d}\right)-(\vec{\omega} \bullet \nabla) \vec{u}_{d}\right] \bullet \hat{n} d A=0
$$

Note that equating the time-derivative of the circulation to zero is a specification, and it is the basis for obtaining a formulation for the diffusion velocity.

\subsection{Determination of the Diffusion Velocity}

Equation (11) implies that the integrand must be zero, or

$$
\frac{\partial \vec{\omega}}{\partial t}=-\left(\left(\vec{u}_{d} \bullet \nabla\right) \vec{\omega}+\vec{\omega}\left(\nabla \bullet \vec{u}_{d}\right)-(\vec{\omega} \bullet \nabla) \vec{u}_{d}\right)
$$

Equating the right hand sides of Eq. (12) and the diffusion equation, Eq. (9), yields an equation for the diffusion velocity in terms of the vorticity field, 


$$
\left(\vec{u}_{d} \bullet \nabla\right) \vec{\omega}+\vec{\omega}\left(\nabla \bullet \vec{u}_{d}\right)-(\vec{\omega} \bullet \nabla) \vec{u}_{d}=-v \nabla^{2} \vec{\omega} .
$$

Using the vector identities, $\quad \nabla \times(\nabla \times \vec{\omega})=\nabla(\nabla \bullet \vec{\omega})-\nabla^{2} \vec{\omega}, \quad \nabla \bullet \vec{\omega}=0$, and $\nabla \times(\vec{u} \times \vec{\omega})=(\vec{\omega} \bullet \nabla) \vec{u}-(\vec{u} \bullet \nabla) \vec{\omega}+\vec{u}(\nabla \bullet \vec{\omega})-\vec{\omega}(\nabla \bullet \vec{u})$, Eq. (13) can be written in a form in which the diffusion velocity appears only once,

$$
-\nabla \times\left(\vec{u}_{d} \times \vec{\omega}\right)=v \nabla \times(\nabla \times \vec{\omega})
$$

This equation implies that,

$$
\vec{u}_{d} \times \vec{\omega}=-v \nabla \times \vec{\omega} .
$$

This equation indicates that if a component of the diffusion velocity exists in the direction of the vorticity vector, then at least one component of the cross product $\vec{u}_{d} \times \vec{\omega}$ is zero. As a result, Eq. (15) yields no information regarding the component of the diffusion velocity in the direction of the vorticity vector. Thus, without further analysis, this formulation provides a diffusion velocity only in two dimensions, since the velocity field is always perpendicular to the vorticity field in two dimensions. We leave the three-dimensional case for future consideration, and restrict further discussions herein to the two-dimensional case.

To find the two-dimensional diffusion velocity, consider the vorticity vector to be aligned with one of the coordinate axes in a rectilinear system with unit vectors $(\hat{i}, \hat{j}, \hat{k})$, and $\vec{\omega}=\left(0,0, \omega_{z}\right)$. Then the components of Eq. (15) are

$$
\begin{aligned}
& u_{d, y}=-\frac{v}{\omega_{z}} \frac{\partial \omega_{z}}{\partial y}, \\
& u_{d, x}=-\frac{v}{\omega_{z}} \frac{\partial \omega_{z}}{\partial x},
\end{aligned}
$$

or using the notion that $\nabla^{\prime}$ indicates the gradient operator in one or two dimensions,

$$
\vec{u}_{d}=-\frac{v}{\omega_{z}} \nabla^{\prime} \omega_{z}
$$

This is the same form of the diffusion velocity as obtained by Ogami and Akamatsu ${ }^{7}$, although they used a different approach which begins with the two-dimensional equations. The fact that the diffusion velocity has vorticity in its denominator is not a problem since in a vorticity method, regions of zero vorticity are not considered. 
We note that the divergence of the diffusion velocity is not solenoidal, in general, as seen from,

$$
\nabla^{\prime} \cdot \vec{u}_{d}=v\left[\left(\frac{\nabla^{\prime} \omega_{z}}{\omega_{z}}\right)^{2}-\frac{\nabla^{\prime 2} \omega_{z}}{\omega_{z}}\right]
$$

Thus, it appears that diffusion can in fact be described as a convective process in which convection occurs at a non-solenoidal diffusion velocity.

\section{Effects of Non-Solenoidal Velocity Fields}

Two effects of convection in a non-solenoidal velocity field are to change the area of a fluid region and to change local vorticity values. We first consider the change in the fluid area as specified kinematically. The definition for the time-rate-of-change of volume of a differential fluid element $\delta V^{2}$

$$
\frac{1}{\delta V} \frac{d \delta V}{d t}=\nabla \bullet \vec{u} .
$$

For two-dimensions, the time-rate-of-change of a differential area $\delta A$ is

$$
\frac{1}{\delta A} \frac{d \delta A}{d t}=\nabla \cdot \vec{u},
$$

and for a one-dimensional differential length $\delta L$,

$$
\frac{1}{\delta L} \frac{d \delta L}{d t}=\nabla \bullet \vec{u} .
$$

We will use this one-dimensional result later when we compare one-dimensional diffusion using this method with an analytical solution.

The effect of the velocity divergence on the vorticity field can be discerned by examining Eq. (12) which is repeated here as

$$
\frac{\partial \vec{\omega}}{\partial t}+\left(\vec{u}_{d} \bullet \nabla\right) \vec{\omega}=-\vec{\omega}\left(\nabla \bullet u_{d}\right) .
$$

An interpretation of this equation is that as vorticity convects at the diffusion velocity (as indicated by the left-hand side of Eq. (22)), the vorticity value changes in proportion to the value of the vorticity and the divergence of the velocity field (as indicated by the righthand side of Eq. (22)). Thus, for example, if $\nabla \bullet \vec{u}_{d}>0$, the magnitude of the vorticity will decrease with time. This is consistent with the diffusion velocity concept in which an 
increase in area for a fixed circulation implies a decrease in vorticity. Next, we implement the non-solenoidal diffusion velocity in a vortex blob algorithm.

\section{Implementation of the Non-Solenoidal Diffusion Velocity Method in a Vortex Blob Method}

The non-solenoidal diffusion velocity can be applied to vortex blobs just as the fluid velocity is applied in discrete vortex methods. Implementation of the diffusion velocity divergence requires some additional development, and is discussed below. First, however, some details of a vortex blob algorithm are presented to provide a framework for the implementation of the diffusion velocity and its divergence.

\section{Details of Vortex Blobs}

A commonly used vortex method represents the vorticity field as a collection of vortex blobs, in which each blob has a known circulation $\Gamma_{j}$, and has a radially symmetric, Gaussian distribution of vorticity about its center $\vec{x}_{j}$. The vorticity field is approximated by the collection of blobs according to

$$
\vec{\omega}(\vec{x})=\sum_{j} \frac{\Gamma_{j}}{\pi \sigma_{j}^{2}} e^{-\left(\frac{\left|\vec{x}_{j}-\vec{x}\right|}{\sigma_{j}}\right)^{2}},
$$

where $\sigma_{j}$ is referred to as the core radius, which is a characteristic length of the area associated with each blob. The choice of a Gaussian vorticity distribution is one of many possible choices, but for our purposes it is useful since core function similarity is maintained if the core size changes.

The velocity field associated with a vorticity field is typically obtained from the Biot-Savart law, but a simpler approach is to use Stokes' theorem

$$
\int_{A} \vec{\omega} \bullet \hat{n} d A=\oint_{C} \vec{u} \bullet d s
$$

where $C$ is the integration path around the area $A$ of the vortex core, with arc length $d s$. Substituting the Gaussian vorticity distribution of a single vortex into the area integral in Eq. (24) yields 


$$
\int_{A} \vec{\omega} \cdot \hat{n} d A=\Gamma_{j}\left[1-e^{-\left(\frac{\left|\hat{x}_{j}-\dot{x}\right|}{\sigma_{j}}\right)^{2}}\right],
$$

which reduces to the circulation of a blob at $\left|\vec{x}_{j}-\vec{x}\right|=\infty$, as required. Since the core function is radially symmetric, the integral on the right-hand side of Eq. (24) (for a single vortex) can be expressed as

$$
\oint_{C} \vec{u} \bullet d s=u_{\theta}(r) 2 \pi r,
$$

where $r=\left|\vec{x}_{j}-\vec{x}\right|$. Equating the right hand sides of Eq. (25) and Eq. (26), transforming the circumferential speed $u_{\theta}$ into cartesian velocity components, and summing the velocity contributions for each vortex gives the often-used expression for the velocity field,

$$
\vec{u}(\vec{x})=\frac{1}{2 \pi} \sum_{j} \frac{\Gamma_{j} \hat{k} \times\left(\vec{x}_{j}-\vec{x}\right)}{\left|\vec{x}_{j}-\vec{x}\right|^{2}}\left[1-e^{-\left(\frac{\left|\vec{x}_{j}-\vec{x}\right|}{\sigma_{j}}\right)^{2}}\right] .
$$

Since vorticity convects inviscidly at the local fluid velocity, the motion of each vortex is specified by

$$
\frac{d \vec{x}_{j}}{d t}=\vec{u}\left(\vec{x}_{j}\right)
$$

For inviscid, incompressible flows, no further development is required since the circulation does not change with time. As a result, the evolution of an inviscid flow is described by integrating Eq. (28) for a given collection of vortices, each with known constant circulations. A vortex blob is assumed to convect as a whole at the velocity of its center $\vec{x}_{j}$, while the size and circular shape of the blob are assumed to remain fixed. We shall make similar assumptions to convect vortex blobs at the diffusion velocity.

For a viscous flow, the vorticity and size of each blou can change with time. These changes are taken into account using the divergence of the diffusion velocity field.

\subsection{Calculation of Diffusion Velocity and Its Divergence}

In order to calculate the viscous diffusion velocity, Eq. (17), the gradient of the vorticity field must be evaluated. To do this, the method proposed by Fishelov ${ }^{4}$ is used, in which 
the gradient of the vorticity field is approximated by applying the gradient operator to the core function in the expression for the approximate vorticity field, Eq. (23). The circulations $\Gamma_{j}$ and core radii $\sigma_{j}$ are not differentiated. Since the contribution by each vortex blob to the vorticity field is radially symmetric about the center of each blob, the gradient operator is simply the derivative with respect to the distance from the center of each blob.

As will be shown, the divergence of the diffusion velocity is an important aspect of the diffusion velocity method, and is given by

$$
\nabla^{\prime} \cdot \vec{u}_{d}=v\left[\left(\frac{\nabla^{\prime} \omega_{z}}{\omega_{z}}\right)^{2}-\frac{\nabla^{\prime 2} \omega_{z}}{\omega_{z}}\right]
$$

Evaluation of Eq. (29, requires the Laplacian of the vorticity field to be approximated in the same way as the gradient of the vorticity field; i.e., by double differentiation of the core function with respect to the distance from the blob center, $\vec{x}_{j}$. Expressions for the two-dimensional gradient and Laplacian of the vorticity field are given in Appendix A.

\subsection{Implementation of the Diffusion Velocity Divergence}

To gain some insight into the physical nature of the diffusion velocity approach, consider a a domain with only a single vortex. At the center of the vortex, the vorticity is $\Gamma_{j} / \pi \sigma^{2}{ }_{j}$, the gradient of the vorticity is zero, and the Laplacian of the vorticity is $-4 \Gamma_{j} / \pi \sigma_{j}^{4 j}$. Since the diffusion velocity is proportional to the gradient of vorticity (see Eq. (17)), and the gradient of vorticity is zero, the diffusion velocity is zero. Thus, the center of the vortex does not move. The divergence of the diffusion velocity for a single vortex can be shown to be $4 v / \sigma_{j}^{2}$

As mentioned earlier, a non-zero divergence changes the area of a fluid element. In terms of vortex blobs, a non-zero divergence is assumed to change the area of the vortex blob. In analogy with the evolution of a fluid area, Eq. (20), the evolution of the characteristic fluid area $\pi \sigma_{j}^{2}$ is,

$$
\frac{1}{\pi \sigma_{j}^{2}} \frac{d\left(\pi \sigma_{j}^{2}\right)}{d t}=\nabla^{\prime} \bullet \vec{u}_{d} .
$$

Implicit in this formulation is the assumption that a vortex blob expands about its center and remains circular. This is consistent with the level of approximation used for inviscid transport in which vortex blobs remain circular and do not change size as they convect.

For a single vortex, which has a divergence of $4 v / \sigma^{2}$,

$$
\frac{d \sigma_{j}^{2}}{d t}=4 \mathrm{v}
$$


which is the well-known result for the diffusion of a two-dimensional Gaussian vorticity distribution. Moreover, it is the same as the so-called diffusing core scheme that, within a vortex method, is known to represent a set of equations that are not the Navier-Stokes equations. This demonstrates that the diffusing core approach is appropriate only for a single vortex. For multiple blobs, the diffusing vortex scheme omits two important features: 1) contributions by other vortices to the divergence and hence the time-rate-ofchange of each blob area, and 2) ilie motion of vortex blobs at the diffusion velocity. Our new scheme includes both convection and expansion due to diffusion.

\section{Summary of the Diffusion Velocity Vorticity Formulation of the Two- Dimensional Navier-Stokes Equations}

In the derivation of the non-solenoidal diffusion velocity method, the two-dimensional Navier-Stokes equations were considered in two simultaneous steps, one step being inviscid transport, the other step being viscous transport. Having derived the non-solenoidal diffusion method under this partitioning, it is now convenient to consider two different steps, one step being translation of vortices at the sum of the inviscid fluid velocity and the diffusion velocity, the other step being the expansion of the vortices due to the diffusion velocity divergence. For a collection of vortices with centers at $\vec{x}_{i}$, the translative step consist" of solving

$$
\frac{d \vec{x}_{i}}{d t}=\vec{u}\left(\vec{x}_{i}\right)+\vec{u}_{d}\left(\vec{x}_{i}\right)
$$

to obtain new blob locations. The fluid velocity $\vec{u}\left(\vec{x}_{i}\right)$ is calculated from Eq. (27), and the diffusion velocity $\vec{u}_{d}\left(\vec{x}_{i}\right)$ is calculated from Eq. (17).

Since the diffusion velocity has a non-zero divergence, the area of the blob must change. Assuming that the circular shape is retained, the core radii $\sigma_{i}$ change according to

$$
\frac{d \sigma_{i}^{2}}{d t}=\sigma_{i}^{2}\left(\nabla^{\prime} \cdot \vec{u}_{d}\left(\vec{x}_{i}\right)\right)
$$

The uivergence of the diffusion velocity is given by Eq. (18). The circulations $\Gamma_{j}$ used to calculate the fluid and diffusion velocities remain constant.

Equations (32) and (33) must be solved simultaneously because the vorticity gradients used to calculate the diffusion velocity and its divergence depend strongly on the core size. Expressions for the gradient and Laplacian of the vorticity field used to calculate the diffusion velocity and its divergence are given in Appendix A. Note that the change in vorticity occurs implicitly as the circulation is held constant and the core size increases. 


\section{Evaluation of Accuracy of the Diffusion Velocity Technique}

To evaluate the accuracy of the diffusion velocity method, a one-dimensional diffusion problem that has an analytical solution is simulated numerically, and the results are compared with the analytical solution.

\section{Problem Statement}

The governing equation for one-dimensional diffusion with constant diffusivity is

$$
\frac{\partial \omega}{\partial t}=v \frac{\partial^{2} \omega}{\partial x^{2}} \text { on }-\infty<x<\infty
$$

We consider the problem which has initial conditions

$$
\begin{aligned}
& \omega=1 \text { on }-h<x<h \\
& \omega=0 \text { elsewhere. }
\end{aligned}
$$

The dimensionless length $x^{*}$ is $x / h$, the dimensionless time $t^{*}$ is $v t / h^{2}$.

\section{Analytical Solution}

The analytical solution to Eq. (34) and Eq. (35) is

$$
\omega(x, t)=\frac{1}{2}\left[\operatorname{erf}\left[\frac{1-x^{*}}{2 \sqrt{t^{*}}}\right]+\operatorname{erf}\left[\frac{1+x^{*}}{2 \sqrt{t^{*}}}\right]\right] .
$$

\section{Diffusion Velocity Simulations of Pure Diffusion}

For the numerical simulation, the vorticity distribution is given by a collection of vortex blobs with one-dimensional Gaussian core functions. The one-dimensional vorticity field, its gradient and Laplacian are given in Appendix B. These expressions are used to calculate the one-dimensional diffusion velocity and its divergence.

By analogy with the evolution of a fluid area, Eq. (21), the evolution of the characteristic fluid area of a one-dimensional vortex blob, $\sqrt{\pi} \sigma_{j}$, is

$$
\frac{1}{\sigma_{j}} \frac{d \sigma_{j}}{d t}=\frac{\partial \vec{u}_{d}}{\partial x}
$$

Four numerical simulations are performed to show the effects of core size and divergence of the diffusion velocity. In each case, the initial vorticity field is represented by 50 vortex 
a)

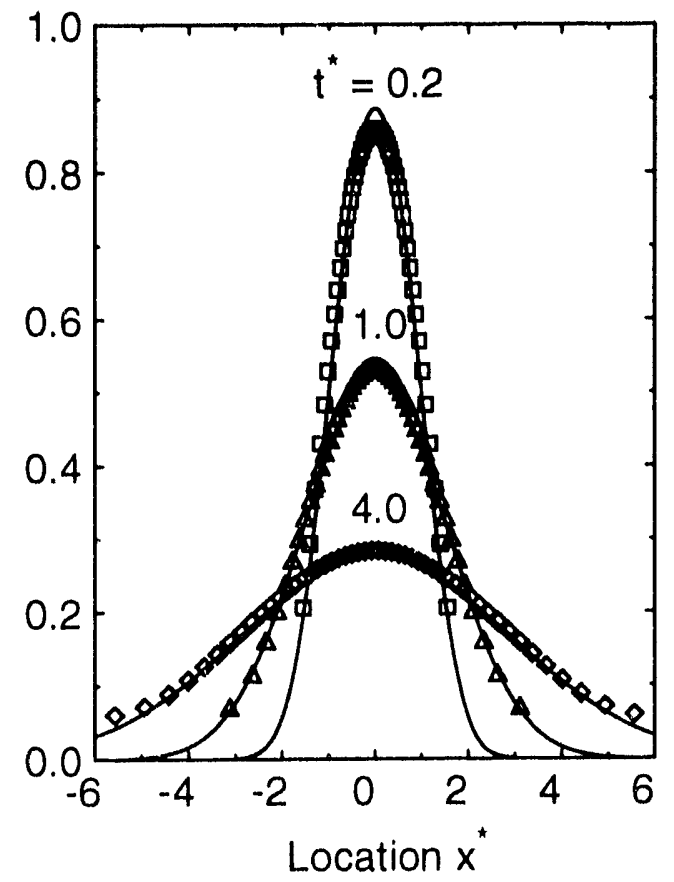

b)

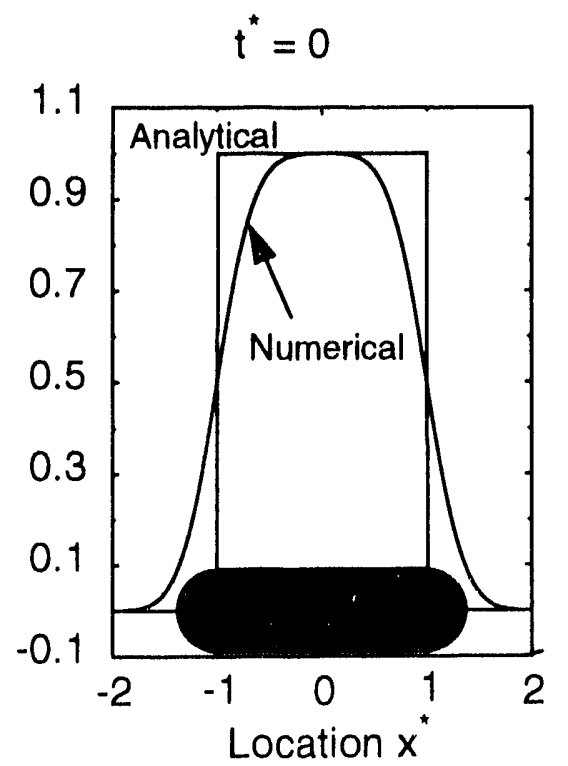

Figure 2 Simulation of diffusion using the diffusion velocity method. Effects of the diffusion velocity divergence are omitted, and the core size is $\sigma / \mathrm{h}=0.4$. a) Comparison of analytical solutions (continuous lines) and diffusion velocity solutions, where symbols denote the location of vortex blobs. Solutions are shown for times of (), (0.2, 1.0, and 4.(). b) The blob representation of the initial vorticity distribution is poor for the large core size, $\sigma / h=() .4$. The core size is shown to scale with respect to the $\mathrm{x}$ axis.

blobs with uniform core sizes which are distributed uniformly in the region $-1<x / h<1$. Initial dimensionless core sizes of $\sigma / h=0.4$ and $\sigma / h=0 .(14$ are considered, and the initial circulations of the vortex blobs have the same value as their respective dimensionless core sizes. These values yield a maximum vorticity of unity in the field.

The equations were integrated with a 3 rd-4th order Runge-Kutta-Fehlberg algorithm with time-step adaption based on a specified relative error criterion of $10^{-6}$. Typical dimensionless time steps are on the order of $\mathrm{O}\left(10^{-2}\right)$, which, as we shall see, depend on whether the divergence of the diffusion velocity field is considered.

The first two cases omit the effects of the divergence of the diffusion velocity for the two core sizes. The latter two cases consider the same two core sizes, but include the effects of divergence of the diffusion velocity. The case of $\sigma / h=0.4$ without considering the divergence was presented by Ogami and Akamatsu and is reproduced in Figure 2 a. Symbols denote the vorticity at blob locations and the solid lines denote the analytical solutions. The numerical results are in good agreement with the analytical solutions, and without further consideration, one might (incorrectly) conclude that it is not necessary to take into account the divergence of the diffusion velocity. Figure $2 b$ shows that the numerical representation of the initial condition is very poor for the large core size used. With such a poor representation of the initial condition, the good agreement at times greater than zero 

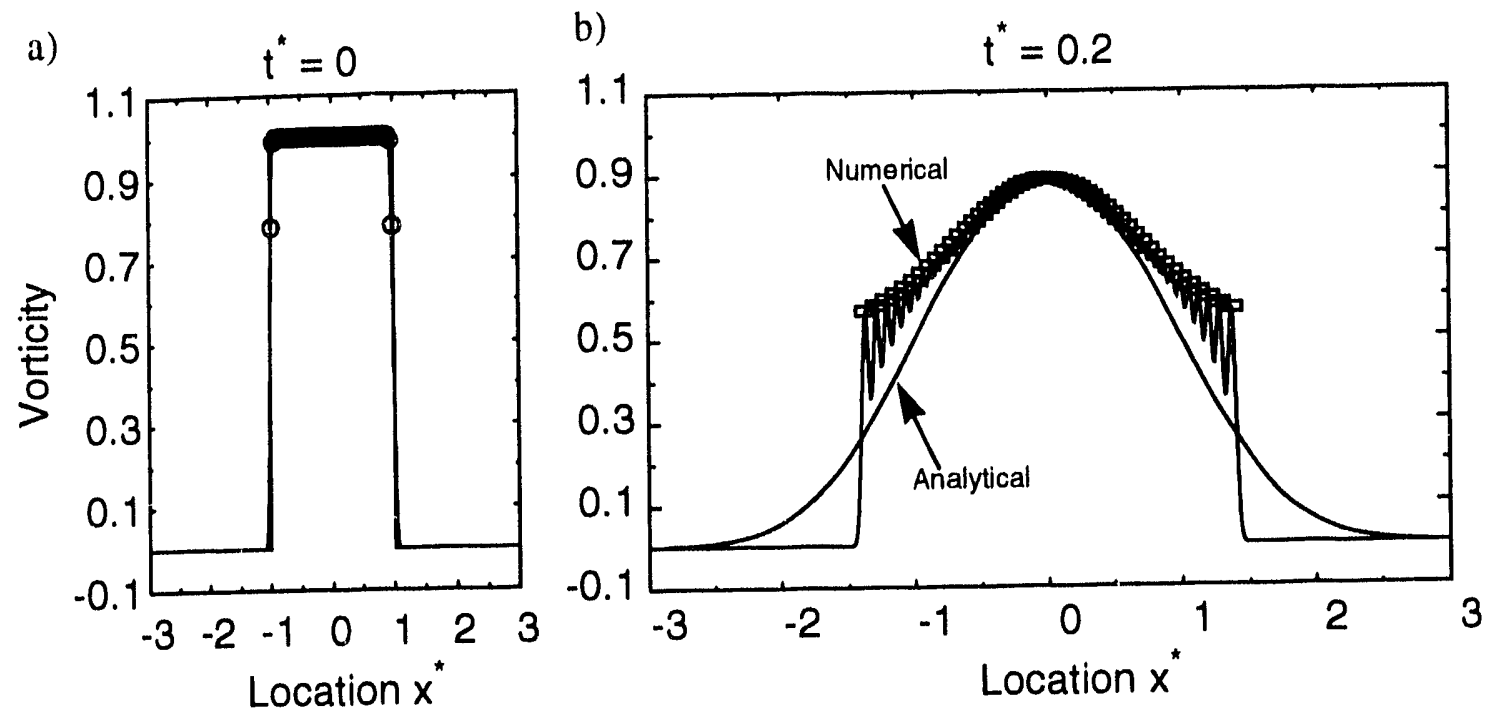

Figure 3 Numerical solution for a core size ten times smaller than the previous simulation. Effects of the diffusion velocity divergence are omitted, and the core size is $\sigma / \mathrm{h}=0.04$. Symbols denote the blob locations. a) The initial condition is well-represented for this core size. b) Large errors are shown as differences between the symbols and the analytical solution. The continuous numerical vorticity distribution contains non-physical "wiggles," due to the omission of diffusion velocity divergence effects.

is, at best, fortuitous. In addition, the cores are highly overlapped, to a degree which is not practical for typical vortex methods. The cores are shown to scale in Figure $2 b$ at $t^{*}=0$. The continuous vorticity distribution shown for the initial condition is obtained by evaluating Eq. (1) in Appendix B at 1200 points distributed uniformly on the interval $-6<x<6$. Ogami and Akamatsu did not present the initial condition.

Next, we consider a core size ten times smaller, $\sigma / h=0.04$, again omitting the effects of divergence to demonstrate the undesirable features of so doing. Even for this smaller core, the overlap between adjacent cores is considerable: the separation between core centers is one core radius, thus cores are overlapped by a full core radius. As shown in Figure $3 a$, the initial condition is represented accurately by the smaller cores. At times greater than zero, however, the vorticity at each blob location is highly inaccurate, as shown in Figure $3 \mathrm{~b}$. Additionally, the implied vorticity distributions (the lines passing through the symbols) contain non-physical "wiggles." The "wiggles" arise because the cores are no longer overlapped, due to the fact that they convect at different velocities. In this case, outer vortices convect faster than inner vortices, so the vortices become separated and the overlap decreases. Core overlap is essential to maintaining smooth vorticity fields, and lack of it results in non-physical "wiggles" such as those shown in Figure $3 \mathrm{~b}$. 
a)

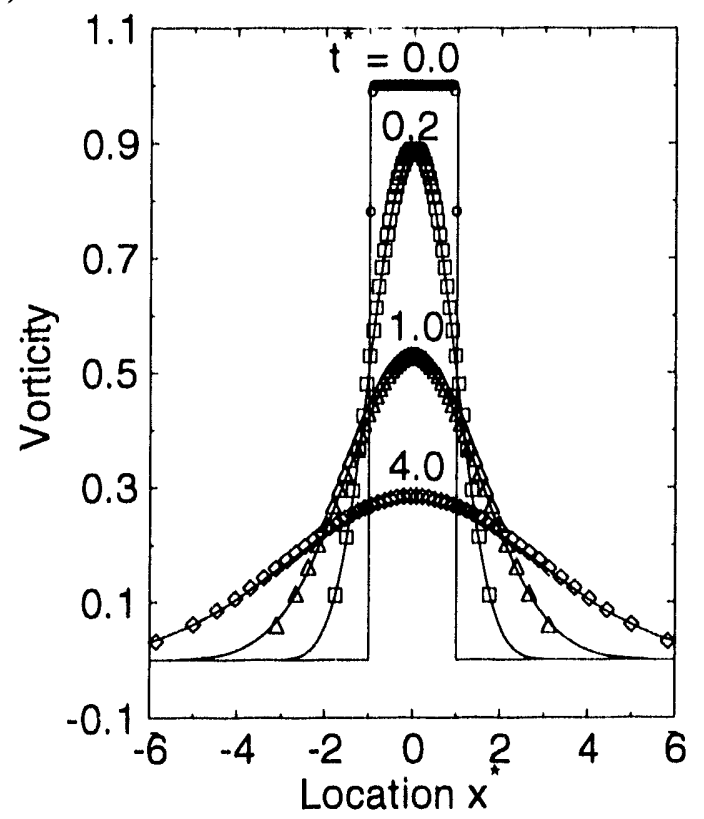

b)

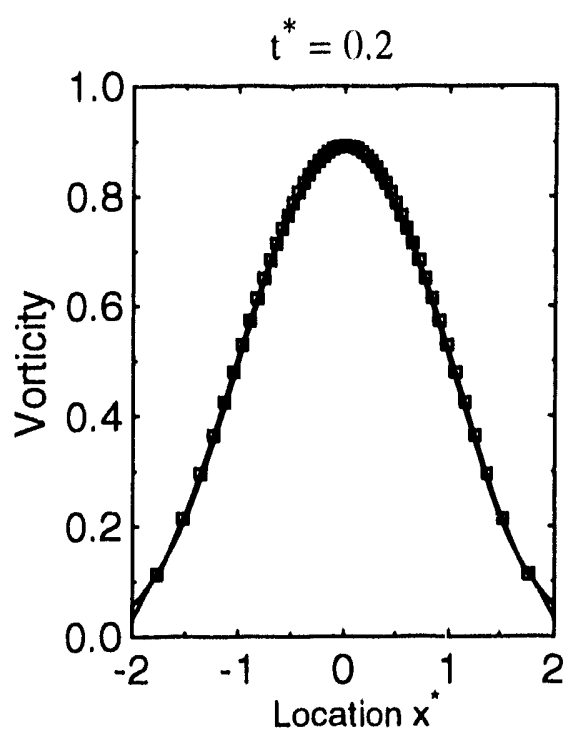

Figure 4 Simulation of diffusion including the effects of the diffusion velocity divergence. The core size is $\sigma / h=0.04$. a) Inclusion of the divergence results in good agreement with the analytical solution. b) Including divergence effects also eliminates wiggles in the numerical vorticity distribution.

The effects of the velocity divergence are now considered for the same small core size, $\sigma / h=(i, 6,4$. Figure $4 a$ shows that including the diffusion velocity divergence greatly improves the accuracy of the diffusion velocity method. Not only is the vorticity at the blob locations accurate, but the continuous vorticity distributions do not contain the "wiggles" seen previously. Figure $4 \mathrm{~b}$ shows the vorticity distribution at the same time as Figure $3 \mathrm{~b}$. "Wiggles" do not occur because the cores are expanding at a rate that is large enough to keep the cores overlapped even as their centers become increasingly separated. At $t^{*}=4$, the solution also remains free of "wiggles," but there is an error of $2.6 \%$ at $x^{*}=0$ (barely perceptible in Figure 4a), which decreases rapidly with distance from the center. This error is appears to be due to the relatively small number of vortices $(50)$ used for these demonstration simulations, and decreases with increasing numbers of vortices. For example, using 200 vortices $(\sigma / h=0.04)$, the maximum error at $t^{*}=4$ is $0.048 \%$, as compared to $2.6 \%$ with 50 vortices. Adaption techniques could also be applied to this problem to assist in maintaining the overlap of vortices that is necessary for high accuracy. 


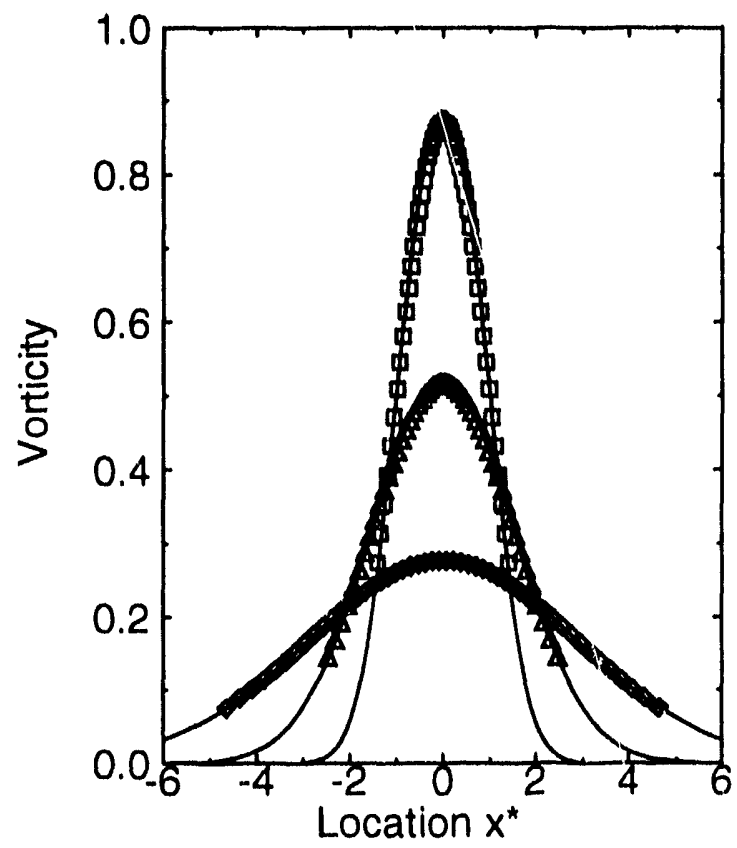

Figure 5 Comparison of results from the non-solenoidal diffusion velocity technique for a core size of $\sigma / \mathrm{h}=0.4$.

The case with the large cores (dimensionless size 0.4 ) including divergence effects is shown in Figure 5. This demonstrates that the non-solenoidal diffusion velocity technique also performs well with large cores. Although, this result has little meaning considering the poor representation of the initial condition with such large cores, as shown in Figure Figure $2 b$.

Returning to the case of the smaller cores $(\sigma / \mathrm{h}=0 .(04)$ where the divergence was included, the values of the divergence and core size are examined. Figure 6 shows the divergence and size of the cores at dimensionless times of 0.2 and 1.(). The expansion of the outermost cores is nontrivial: they expand by a factor of approximately six at $t^{*}=0.2$ and ten at $t^{*}=1$. At very early times, only the outermost cores have significant velocities. This situation gives rise to large divergences at the outermost vortices, which caluses them to expand, while the other vortices remain relatively constant in size. As time proceeds, the velocities of the cores become more uniform, yielding a smaller and more uniform distribution of the divergence, so that the cores expand uniformly. The outer cores, however, always remain larger than inner cores, since they are the only ones to experience the large expansion rate at early times.

The simulations presented above indicate that including the divergence of the diffusion velocity is essential to obtaining an accurate simulation of diffusion. Care must be taken in this approach, however, since a new time scale is introduced when the divergence is taken into account. The new time scule is the inverse of the divergence, and is manifested in the evolution of the core sizes, e.g., Eq. (37). At early times, when the divergence is largest, stability and accuracy of the time integration require significantly smaller time-steps. For 

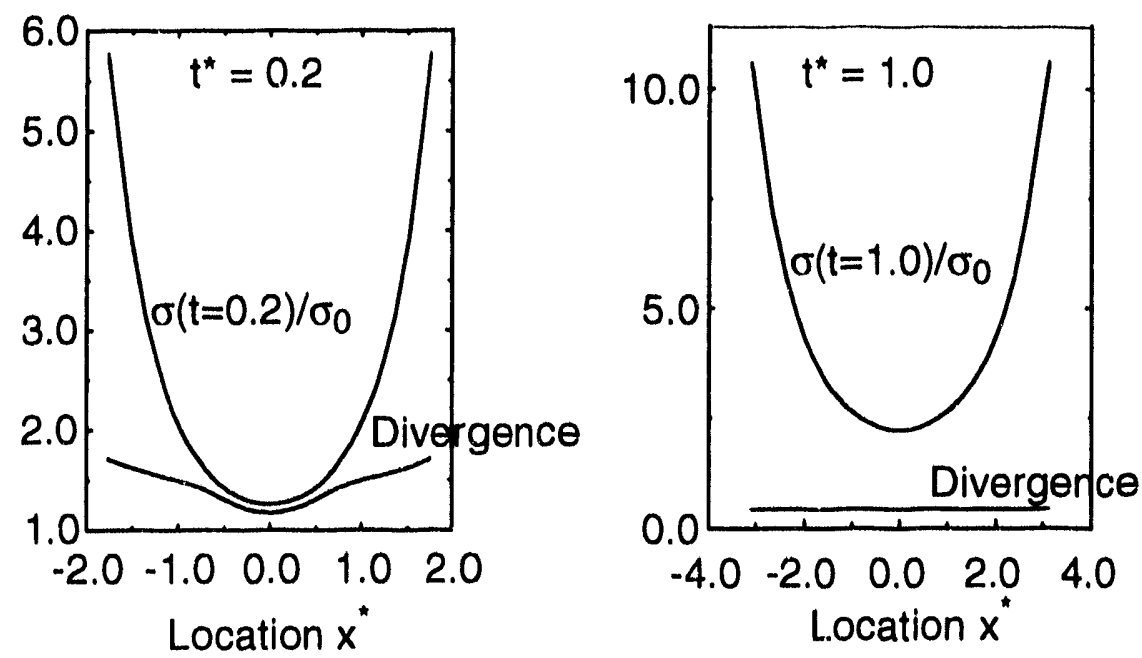

Figure 6 Distribution of core size and divergence of the diffusion velocity at dimensionless times of 0.2 and 1.0. At $t^{*}=0.2$, the cores at the edges of the field have expanded by a factor of approximately 6 , whereas the cores in the center have expanded by less than a factor of two. At $t^{*}=1.0$, the outer cores have expanded by a factor of ten. In each figure, the initial dimensionless core size is $\sigma_{0}=0.04$. The divergence has units of inverse dimensionless time.

example, if divergence effects are omitted, typical dimensionless time-step sizes are $\mathrm{O}\left(10^{-2}\right)$ and "wiggly" solutions are obtained. In order to obtain smooth solutions by including divergence effects, time-steps must be an order of magnitude smaller, $\mathrm{O}\left(10^{-3}\right)$. (These results are based on the restrictive specification that relative errors be less than $\left.10^{-6}\right)$. Thus, for situations where large gradients of vorticity are anticipated (such as flows with large Reynolds numbers), use of an adaptive time integration scheme (such as we used) becomes essential to obtain accurate results.

\section{Discussion}

The comparison of the analytical diffusion solution with results from the non-solenoidal diffusion velocity method indicates that diffusion can be accurately approximated as a convective process. To provide a physical motivation for the treatment of diffusion as a convective process, a Monte Carlo simulation of vorticity diffusion is discussed and related to the non-solenoidal diffusion velocity method.

In a Monte Carlo simulation, the vorticity field would be represented by a collection of particles each with the same vorticity, which does not vary with time or space. The vorticity at a point in space (at any time) would be obtained from a volume-average of the particles in a small volume enclosing the spatial point. Thus, any vorticity distribution can be specified in terms of the particle population density (particles per volume). Diffusion of 
the vorticity field would be simulated by applying a sequence of Gaussian random walks to the particles, and finding local volume averages at points of interest to determine the vorticity as a function of time.

Now consider a particular set of particles and the simply connected volume that contains them. As time advances, the particles become more separated and the volume of the region containing them expands. Since the amount of vorticity associated with the set of particles is constant, the expansion of the volume which encloses the particles amounts to a reduction in the volume-averaged vorticity for that set of particles. Also, for a spatially nonuniform distribution of particles, the center of the volume will move. Thus, diffusion phenomena for a set might be represented as a random walk of the set as a whole in addition $t)$ an expansion of the set.

The volume which encloses a set of points is not a physical volume, since it can overlap the volume of other sets of points. Similarly, vortex blobs can overlap, and as noted by Sarpkaya", "blobs must be regarded as mathematical artifices (many souls in one body!) since vorticity-carrying non-deforming fuid particles cannot occupy the same space at the same time (the exclusion principle)."

In discrete vortex methods, the constant circulation of a finite vortex is analogous to the constant amount of vorticity associated with a particular set of particles. By applying a random walk to the motion of a vortex as a whole, the diffusive expansion of that vortex is not resolved. Since the standard deviation of a random walk variable in each direction varies as $R e^{-1}$ ( $R e$ is the Reynolds number), the expansion rate varies as $R e^{-n / 2}$, where $n$ is the dimension of the problem, $n=1,2$, or 3. Thus, for low Reynolds numbers, diffusive expansion is more important than at high Reynolds numbers. As a result, the random walk approach is not appropriate at low Reynolds numbers. For large Reynolds numbers, where diffusive expansion is smaller and better approximated as being zero, convergence can only be expected in a mean sense since the number of vortices that is computationally affordable is insufficient to obtain statistical convergence.

\section{Summary and Conclusions}

The non-solenoidal diffusion velocity method has been derived for two-dimensional vortex methods. In this method, diffusion is cast as a velocity at which vortices convect with fixed circulation, just as in inviscid vortex methods. The size of each core changes according $t)$ the divergence of the diffusion velocity, and an algorithm to implement this core variation in a vortex blob method was also presented. The inclusion of viscous diffusion effects allows vortex methods to be applied to flows with general finite Reynolds numbers, thus greatly extending their range of application.

This non-solenoidal diffusion velocity method is more accurate than previously developed methods, since previous methods typically considered either the translation or the expansion, when both are necessary to be accurate. The diffusion velocity proposed by Ogami 
and Akamatsu is used to translate vortices, and the model for the core expansion is based on the kinematics of fluid areas in a non-solenoidal velocity field. The accuracy of this method was demonstrated by comparison with an analytical diffusion solution for a range of corc sizes that spans the range of values typically used in vortex calculations.

Our future plans include a brief study to determine if it is necessary to include the effects of all the vortices in calculating the diffusion velocity and its divergence. Preliminary indications are that the diffusion velocity at a particular vortex can be accurately computed by using only a few of the surrounding vortices, rather than all the vortices. This would greatly speed-up the algorithm. We also plan to examine the extension of this approach to three-dimensional flows.

\section{Acknowledgment}

This work was supported by the U. S. Department of Energy under contract DE-AC0476DP()(178\%. We thank Steve Rottler, Roy Baty, Bill Ashurst, and Lou Gritzo for their careful reviews of this work.

\section{References}

${ }^{1}$ Ashurst, W. T., "Numerical Simulation of Turbulent Mixing Layers via Vortex Dynamics," in Turbulent Shear Flows 1., (eds. F. Durst, et al.), Springer-Verlag, pp. 4(1)2413. (Also SAND 77-8612, Sandia Laboratories Report).

${ }^{2}$ Batchelor; G. K., An Introduction to Fluid Mechanics, Cambridge University Press, 1967.

${ }^{3}$ Chorin, A. J., "Numerical Study of Slightly Viscous Flow," J. Fluid Mech., vol. 57, pp. $785-796,1973$.

${ }^{4}$ Fishelov, D., "A New Vortex Scheme for Viscous Flows," J. Comput. Phys., vol. 86, pp. $211-224,1990$.

${ }^{5}$ Greengard, C., "The Core Spreading Vortex Method Approximates the Wrong Equation," J. Comput. Phys., vol. 61, p. 345, 1985.

6Leonard, A., "Vortex Methods for Flow Simulation," J. Comput. Phys., vol. 37, pp. 289335,1980 .

${ }^{7}$ Ogami, Y. and T. Akamatsu, "Viscous Flow Simulation using the Discrete Vortex ModelThe Diffusion Velocity Method," Computers \& Fluids, vol. 19, pp. 433-441, 1991.

${ }^{8}$ Saffman, P. G. and G. R. Baker, "Vortex Interactions," Ann. Rev. Fluid Mech., vol. II, pp. 95-122. 
${ }^{9}$ Sarpkaya, T., "Computational Methods with Vortices," The 1988 Freeman Scholar Lecture, J. Fluids Engineering, vol. 111, no. 1, March, 1989.

${ }^{10}$ Sethian, J. A., and A. F. Ghoniem, "Validation Study of Vortex Methods," J. Comput. Phys., vol. 74, pp. 283-317, 1988. 


\section{Appendix A \\ Formulas for a Two-Dimensional Vorticity Field, its Gradient and Laplacian}

The vorticity field specified by a collection of vortex blobs, each of which has a radially symmetric, Gaussian vorticity distribution, is

$$
\vec{\omega}(\vec{x})=\sum_{j} \frac{\Gamma_{j}}{\pi \sigma_{j}^{2}} e^{-\left(\frac{\left|x^{\prime}-\vec{x}\right|}{\sigma_{j}}\right)^{2}}
$$

The circulation of each blob is $\Gamma_{j}$ and the core radius is $\sigma_{j}$. The gradient of the vorticity field is,

$$
\nabla \vec{\omega}(\vec{x})=-\frac{2}{\pi} \sum_{j} \frac{\Gamma_{j}\left(\vec{x}-\vec{x}_{j}\right)}{\sigma^{4}} e^{-\left(\frac{\left|\vec{x}-\vec{x}_{j}\right|}{\sigma_{j}}\right)^{2}} .
$$

where the circulations and core radii are not differentiated. Similarly, the Laplacian of the vorticity field is,

$$
\nabla^{2} \vec{\omega}(\vec{x})=\frac{4}{\pi} \sum_{j} \frac{\Gamma_{j}}{\sigma_{j}^{4}}\left[\left(\frac{\vec{x}-\vec{x}_{j}}{\sigma_{j}}\right)^{2}-1\right] e^{-\left(\frac{\left|\vec{x}-\vec{x}_{j}\right|}{\sigma_{j}}\right)^{2}}
$$




\section{Appendix B}

\section{Formulas for a One-Dimensional Vorticity Field, its Gradient and Laplacian}

A one-dimensional distribution of vorticity described by a collection of vortex blobs each of which has a Gaussian vorticity distribution is given by,

$$
\vec{\omega}(x)=\sum_{j} \frac{\Gamma_{j}}{\sqrt{\pi} \sigma_{j}} e^{-\left(\frac{\left|x-x_{j}\right|}{\sigma_{j}}\right)^{2}} .
$$

The circulation of each blob is $\Gamma_{j}$ and the core radius is $\sigma_{j}$. The gradient of the vorticity field is,

$$
\nabla \vec{\omega}(x)=-\frac{2}{\sqrt{\pi}} \sum_{j} \frac{\Gamma_{j}\left(x-x_{j}\right)}{\sigma_{j}^{3}} e^{-\left(\frac{x-x_{j}}{\sigma_{j}}\right)^{2}}
$$

where the circulations and core radii not differentiated. Similarly, the Laplacian of the vorticity field is,

$$
\nabla^{2} \vec{\omega}(x)=\frac{2}{\sqrt{\pi}} \sum_{j} \frac{\Gamma_{j}}{\sigma_{j}^{3}}\left[2\left(\frac{x-x_{j}}{\sigma_{j}}\right)^{2}-1\right] e^{-\left(\frac{x-x_{j}}{\sigma_{j}}\right)^{2}}
$$




\section{Distribution}

Analytical Methods (2)

P.O. Box 3786

Bellevue, WA 98009

Attn: F. Dvorak

B. Maskew

Prof. Holt Ashley

Dept. of Aero. and Astronautics

Stanford University

Stanford, CA 94305

Mr. James Fein

Code 1221

ONR

800 N. Quincy St.

Arlington, VA 22217

Dr. H. Higuchi

Mech. Eng. \& Aero. Dept

Syracuse University

Syracuse, NY 13244

Capt. Hank Helin

HQ USFA/DFAN

USAF Academy, CO 80840-5701

Mr. John D. Johnson

Code 324

NAWC

China Lake, CA 93555-6001

Mr. Gary W. Jones

DARPA

Submarine Technology

1400 Wilson Blvd.

Arlington, VA 22209-2308

Prof. Joseph Katz

Dept. Aerospace Eng. and Eng. Mech.

San Diego State University

San Diego, CA 92115

Dr. George A. Keramidas

Code 4420

NRL

Washington, DC 20375
Prof. A. Leonard

Graduate Aeronautics Lab.

California Institute of Technology

Pasadena, CA 91125

Prof. M. Luttges

Dept. of Aerospace Eng. Sci.

University of Colorado

Boulder, CO 80309-0429

Dr. R. C. Maydew

5305 Queens Ct. NE

Albuquerque, NM 87109

Prof. R. N. Meroney

Dept. of Civil Eng.

Colorado State University

Fort Collins, CO 80521

NASA Johnson Space Center (2)

Attn: EG3

Houston, TX 77058

Attn: D. B. Kanipe

R. E. Meyerson

New Mexico State University (2)

Dept. of Mech. Eng.

Las Cruces, NM 88003

Attn: Ron Pederson

G. Reynolds

Mr. W. J. Quinlan

Aerodynamic Design

Ford Motor Company

21175 Oakwood Blvd.

Dearborn MI, 48123

Prof. T. Sarpkaya

Dept. Mech. Eng.

Code 69-SL

Naval Postgraduate Academy

Monterey, CA 93943

Prof. Roger L. Simpson

Dept. Aerospace and Ocean Eng. Virginia Polytechnic Institute and State University

Blacksburg, VA 24061 
Mr. J. T. Strickland

Sterling Chemical Co.

Post Office Box 1311

Texas City, TX 77592

Texas Tech University (2)

Dept. of Mech. Eng.

Lubbock, TX 79409

Attn: J. H. Lawrence

$$
\text { J. W. Oler }
$$

University of New Mexico (2)

Dept. of Mech. Eng.

Albuquerque, NM 87106

Attn: M. S. Ing her

C. R. Truman

Dr. James Uhlman

Code 804, Bldg. 108/2

Naval Undersea Warfare Center

Newport, RI 02841-5047

U. S. Army, Natick (9)

RD\&E Center

AMED/ETD

Kansas St.

Natick, MA 01760

Attn: John Caligeros

Maurice Gionfriddo

Steven Kunz

Calvin Lee

Andrew Mawn

Robert Rodier

James Sadeck

Earl Steeves

Gary Thibault

Prof. R. E. Wilson

Dept. Mech. Eng.

Oregon State University

Corvallis, OR 97331
G. F. Homicz

S. N. Kempka (10)

C. C. Wong

A. C. Ratzel

M. R. Baer

J. R. Torczynski

R. D. Skocypec

S. Tiesczen

W. P. Wolfe

C. E. Hailey

R. S. Baty

J. H. Strickland (15)

W. L. Hermina

W. H. Rutledge

W. L. Oberkampf

H. S. Morgan

R. K. Thomas

G. A. Laguna

G. N. Beeler

Technical Library (5)

Doc.Proc.DOE/OSTI (10)

Technical Publication (1)

J. C. Cummings

G. R. Otey

W. C. Nickell

D. F. Wolf

P. C. Klimas

H. M. Dodd

D. E. Berg

M. E. John

R. J. Detry

C. M. Hartwig

R. J. Kee

W. T. Ashurst

Central Tech Files

C. M. Hart

M. M. Newsom

E. G. Kadlec

J. M. Macha

R. L. Woodfin

R. B. Asher 

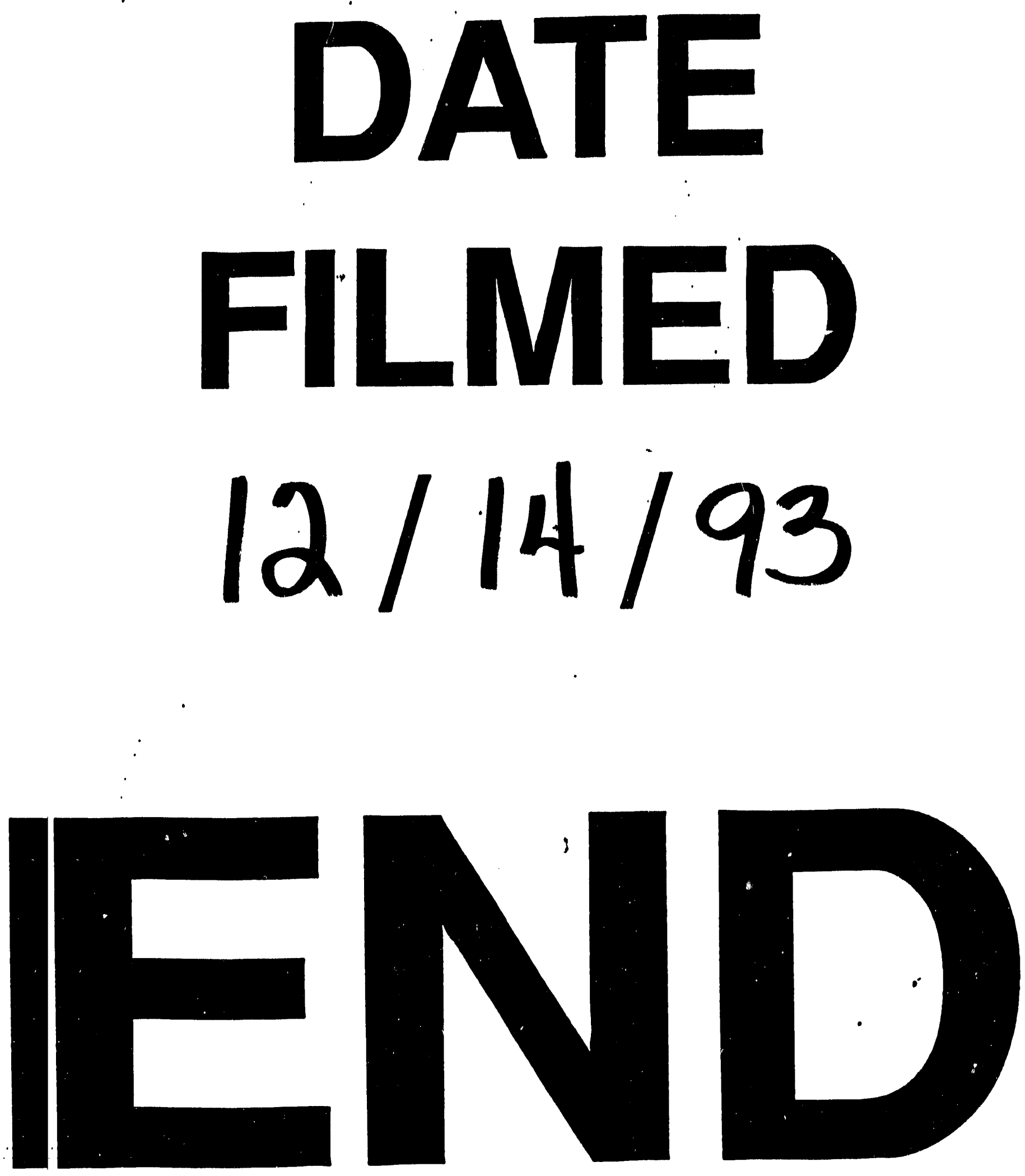
